\begin{tabular}{|c|l|}
\hline Title & Statistical properties of weighted complex networks characterized by metaweights \\
\hline Author(s) & Furuya, Shuhei; Y akubo, Kousuke \\
\hline Citation & $\begin{array}{l}\text { PhysicaA : Statistical Mechanics and its A pplications, 389(6), 1265-1272 } \\
\text { https://doi.org/10.1016/.physa.2009.12.001 }\end{array}$ \\
\hline Issue Date & 2010-03-15 \\
\hline Doc URL & http://hdl.handle.net/2115/42676 \\
\hline Type & article(author version) \\
\hline File Information & PhyA 389-6_1265-1272.pdf \\
\hline
\end{tabular}

Instructions for use 


\title{
Statistical properties of weighted complex networks characterized by metaweights
}

\author{
Shuhei Furuya, Kousuke Yakubo \\ Department of Applied Physics, Hokkaido University, Sapporo 060-8628, Japan
}

\begin{abstract}
We propose several characterizations of weighted complex networks by incorporating the concept of metaweight into the clustering coefficient, degree correlation, and module decomposition. These incorporations make it possible to describe weighted networks depending on how strongly we emphasize weights. Using some applications to real-world weighted networks, we demonstrate that the proposed approach provides rich information that was inaccessible by previous analyses such as the degree correlation for a specific magnitude of weights or the community structure under controlling the importance of roles of the topology and weights.
\end{abstract}

Keywords: Complex network, Statistical analysis, Clustering coefficient, Degree correlation, Community structure PACS: 89.75Hc, 89.75.Fb, 05.10.Gg

\section{Introduction}

The rapid development of computer networks and the computational management of large databases enables us to treat a set of elements and their relations in the world as a complex network that is constructed from many nodes and edges. Complex networks are characterized by many statistical quantities and concepts. The clustering coefficient introduced by Watts and Strogatz [1] represents a measure of how completely the neighbors of a node are interconnected, and it plays an important role in describing the density or small-world property of a network. It is also important to understand

Email addresses: s-furuya@eng.hokudai.ac.jp (Shuhei Furuya), yakubo@eng.hokudai.ac.jp (Kousuke Yakubo) 
the degree correlation, namely, the averaged degree $k_{n n}(k)$ of the neighboring nodes of nodes with the degree $k$ [2], where the degree is the number of edges connected to a node. The attractive or repulsive tendency between hubs (nodes with large degrees) often reflects network formation mechanisms. Some complex networks can be regarded as aggregates of partial communities (groups) that are weakly connected to each other. To determine how a network is constructed from such modules is crucial not only for understanding the structure of the network but also for elucidating functions of the network $[3,4,5,6]$.

In most real-world networks, edges (or nodes) are not equivalent and some quantities (weights) are assigned to them. Recently, networks with weighted edges have been extensively studied because network properties are strongly affected by the distribution of weights in the network [7, 8, 9]. Thus far, various quantities used for characterizing binary networks have been straightforwardly extended for weighted networks. Standard extensions of statistical quantities to weighted networks emphasize structures formed by strong edges (with large weights). However, this is not sufficient for describing the nature of weighted networks. Let us consider two weighted networks. Both networks have the same topological structure and the same distribution of large weights, however the distributions of small weights are different. Statistical quantities dominated by strong edges cannot distinguish these two networks. Therefore, it is necessary to characterize weighted networks by emphasizing a specific magnitude of weights. In our previous paper [10], we introduced metaweights defined as the $q$ th power of weights, where $q$ is a real continuous parameter. When $q$ is a large positive value, strong weights are emphasized more strongly. We can study the topological aspects of a weighted complex network by choosing $q=0$. For a negative $q$, structures constructed by weak edges are selectively emphasized. Using metaweights, the scale-free property [11] of weighted networks has been studied and the $q$ dependence of the scale-free exponent has been obtained [10].

In this study, we propose several characterizations of weighted complex networks by incorporating the concept of metaweight into several statistical quantities. The metaweighted clustering coefficient, metaweighted degree correlation, and module structure based on metaweights are calculated for US airport networks and a scientific collaboration network. From these examples, we demonstrate how our analyses extract detailed information about weighted complex networks. 


\section{Metaweighted clustering coefficient}

Many complex networks have a tendency that two nodes connected to a common node are linked to each other. In order to characterize this tendency statistically, the clustering coefficient $C_{i}$ of a node $i$ is defined as the ratio of the number of connected neighboring nodes of $i$ to the number of all neighboring node pairs $[1,12,13,14,15]$. This quantity has been extended to weighted networks. There exist several definitions of the weighted clustering coefficient $C_{i}^{w}[8,15,16,17,18,19]$. In these definitions of $C_{i}^{w}$, however, only cluster structures made of neighboring nodes connected to the node $i$ by large weights are enhanced. It is also important to clarify whether weakly (or very strongly) linked neighbors form clusters. By introducing metaweights, one can evaluate such details of network properties. In this paper, we define the metaweighted clustering coefficient $C_{i}^{w}(q)$ by extending the weighted clustering coefficient proposed by Barrat et al. [8]. The quantity $C_{i}^{w}(q)$ is given by

$$
C_{i}^{w}(q) \equiv \frac{1}{s_{i}(q)\left(k_{i}-1\right)} \sum_{j, l} \frac{w_{i j}^{q}+w_{i l}^{q}}{2} a_{i j} a_{j l} a_{l i},
$$

where $k_{i}$ is the degree of the node $i, w_{i j}$ is the weight allocated to the edge $i-j, a_{i j}$ is the $(i, j)$ element of the binary adjacency matrix, and $s_{i}(q)$ is the metastrength defined by $s_{i}(q)=\sum_{j} a_{i j} w_{i j}^{q}$. The metaweighted clustering coefficient $C^{w}(q)$ of the entire network is defined as the average of $C_{i}^{w}(q)$ over $i$, i.e., $C^{w}(q)=\sum_{i}^{N} C_{i}^{w}(q) / N$. If $C^{w}(q<0)$ is larger than $C^{w}(q>$ $0)$, the network is densely connected mainly by weak edges and sparsely connected by strong edges, and vice versa. Therefore, the analysis by using the metaweighted clustering coefficient extracts detailed relations between the network topology and the weight distribution.

In order to demonstrate the usefulness of the analysis based on $C^{w}(q)$, we calculate metaweighted clustering coefficients for a US airport network and a scientific collaboration network. The US airport network (USAN-I) obtained from [20] is a weighted network including 500 airports (nodes) in the United States [21]. In this network, a weight represents the number of available seats on a given connection on an yearly basis [8, 22]. Figure 1(a) shows $C^{w}(q)$ rescaled by the topological clustering coefficient $C^{w}(q=0)$. For positive $q$ 's, $C^{w}(q) / C^{w}(0)$ is larger than unity, implying that the network is more densely clustered by strong edges than that of the topological 
(a)

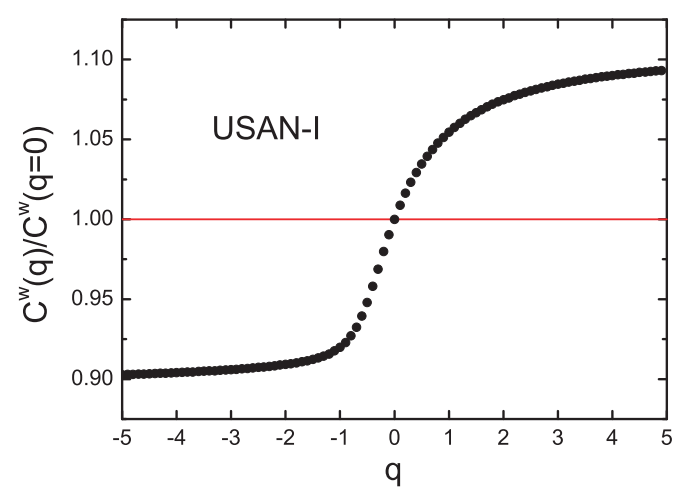

(b)

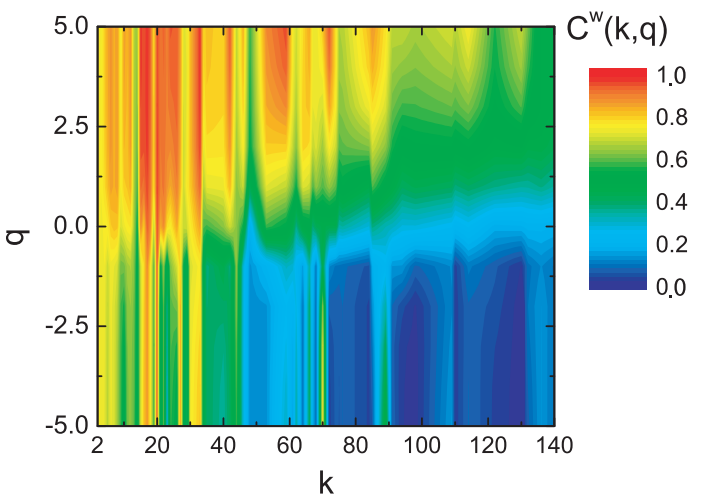

Figure 1: (a) Rescaled metaweighted clustering coefficient for the USAN-I as a function of the parameter $q$. (b) Metaweighted clustering coefficient spectrum for the USAN-I as a function of the degree $k$ and the parameter $q$.

structure. On the contrary, the result for $q<0$ implies that the network is loosely connected by weak edges as compared to the cluster structure of the network topology. This is reasonable because arterial airlines (strong edges) from an airport connect to hub airports $(k \gtrsim 100)$, and hub airports are always interconnected. On the other hand, feeder airlines (weak edges) from an airport connect to local airports $(k \lesssim 100)$ that are not connected to each other. In order to elucidate the relation between cluster structures and weights in more detail, we examine the metaweighted clustering coefficient spectrum $C^{w}(k, q)$ defined by

$$
C^{w}(k, q)=\frac{1}{n_{k}} \sum_{i} C_{i}^{w}(q) \delta_{k k_{i}}
$$

where $n_{k}=\sum_{i} \delta_{k k_{i}}$. Figure $1(\mathrm{~b})$ shows $C^{w}(k, q)$ for the USAN-I. The fact that $C^{w}(k, q)$ is large for $q>0$ and small for $q<0$ and $k \gtrsim 100$ is equivalent to the result shown in Fig. 1(a). Roughly speaking, the metaweighted clustering coefficient spectrum $C^{w}(k, q)$ is a decreasing function of $k$ for $q<0$ and $k \lesssim 100$. Neighboring airports of a very local airport $(k \lesssim 10)$ are hub airports even if edge weights are small. Since these neighboring hub airports are connected to each other, $C^{w}(k \lesssim 10, q<0)$ is large. On the contrary, airports that are weakly connected to a middle-scale airport $(k \sim 50)$ can be local airports, and these may not always be connected to each other. Thus, 
(a)

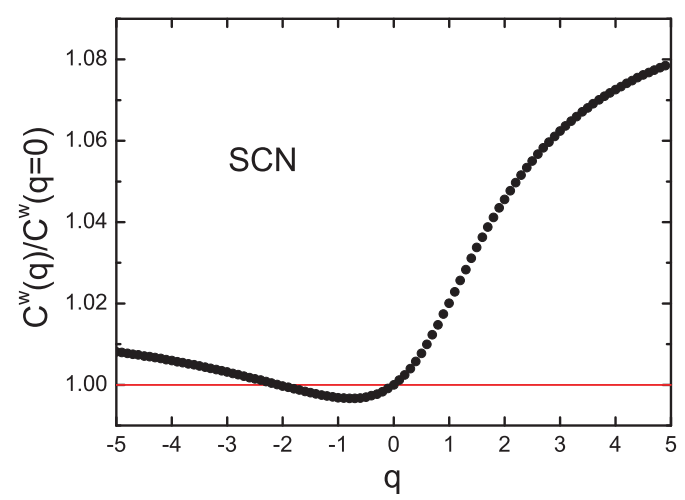

(b)

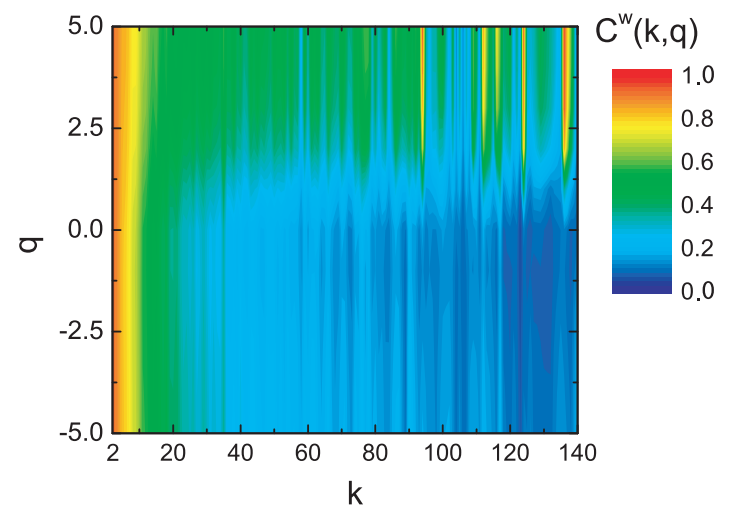

Figure 2: (a) Rescaled metaweighted clustering coefficient for the SCN as a function of the parameter $q$. (b) Metaweighted clustering coefficient spectrum for the SCN as a function of the degree $k$ and the parameter $q$.

$C^{w}(k \sim 50, q<0)$ is smaller than $C^{w}(k \lesssim 10, q<0)$.

The scientific collaboration network (SCN) $[23,24]$ treated in this paper is constructed by 40,421 authors as nodes in the research area of condensed matter physics. Two nodes are connected if the corresponding authors have co-authored paper(s) together. The weight on the edge $i-j$ is defined as $w_{i j}=\sum_{\alpha} \eta_{i \alpha} \eta_{j \alpha} /\left(m_{\alpha}-1\right)$, where $\eta_{i \alpha}=1$ if the author $i$ is an author of the paper $\alpha, \eta_{i \alpha}=0$ otherwise, and $m_{\alpha}$ is the number of co-authors of the paper $\alpha$ [25]. Figure 2(a) shows the $q$ dependence of the rescaled metaweighted clustering coefficient for the SCN. The result depicts that this network has a clusterization tendency by strong edges, whereas by weak edges, it is similar to the topological one. This tendency can be more clearly shown by the metaweighted clustering coefficient spectrum. The spectrum $C^{w}(k, q)$ shown in Fig. 2(b) indicates that authors who are strongly connected to an author tend to be connected to each other as compared to the case in which authors are weakly connected to an author, i.e., $C^{w}(k, q>0)>C^{w}(k, q<0)$. In addition, $C^{w}(k, q)$ is a decreasing function of $k$ for $q<0$. This implies that authors collaborating with a big-name scientist (hub) do not collaborate with each other if the co-author relationship is weak. The metaweighted clustering coefficient $C^{w}(k, q)$ for $q \sim 5$ and $k \gtrsim 50$ is large. Since authors strongly connected to a big-name scientist are also big name as will be shown in Fig. 4, this feature of $C^{w}(k, q)$ implies that big-name scientists collaborating 
tightly with a big-name author are well connected to each other. In both Figs. 1(b) and 2(b), fluctuations of $C^{w}(k, q)$ in the $k$-axis increase with $|q|$. This is because fluctuations at $q=1$ are enhanced as $|q|$ increases, and these are not related to statistical properties of a given network. These enhanced fluctuations lead to a gradual increase in the rescaled coefficient $C^{w}(q) / C^{w}(0)$, shown in Fig. 2(a), as $|q|$ increases in the region of $q<-2$. From these results, one can obtain detailed information about the correlation between strengths of edges and the network topology by introducing the metaweighted clustering coefficient.

\section{Metaweighted degree correlation}

In this section, we argue the degree correlation of a weighted network by introducing metaweights. The assortativity, that is, the tendency of hub nodes to connect to other hubs, of a binary network is characterized by examining the degree correlation between the degree $k$ of a node in the network and the average degree $k_{n n}$ of its neighboring nodes [26, 27, 28, 29, 30]. The average nearest-neighbor degree $k_{n n}$ as a function of $k$ is given by

$$
k_{n n}(k)=\frac{1}{n_{k}} \sum_{i, j} a_{i j}\left(\frac{k_{j}}{k_{i}}\right) \delta_{k_{i} k},
$$

where $a_{i j}$ and $n_{k}$ are defined in Section 2. From this definition and its extension to the weighted average nearest-neighbor degree proposed by Barrat et al. [8], we can straightforwardly define the metaweighted average nearestneighbor degree (MANND) $k_{n n}^{w}(k, q)$ as

$$
k_{n n}^{w}(k, q) \equiv \frac{1}{n_{k}} \sum_{i, j} a_{i j}\left[\frac{w_{i j}^{q} k_{j}}{s_{i}(q)}\right] \delta_{k_{i} k} .
$$

Using this quantity by varying the parameter $q$, the nearest-neighbor degree correlation for only weak or strong connections can be detected.

Here, we illustrate the efficiency of the analysis of metaweighted degree correlations by computing $k_{n n}^{w}(k, q)$ for the previous two examples, namely, the USAN-I and the SCN. Figure 3 shows the result for the USAN-I. We can extract the following facts from this result. (1) The MANND is very small $\left[k_{n n}^{w}(k, q) \lesssim 20\right]$ for $q<0$ and $k \gtrsim 50$, (2) the quantity $k_{n n}^{w}(k, q)$ is a decreasing function of $k$ (disassortative) for $q<0$ and $k \lesssim 50$, (3) the network 


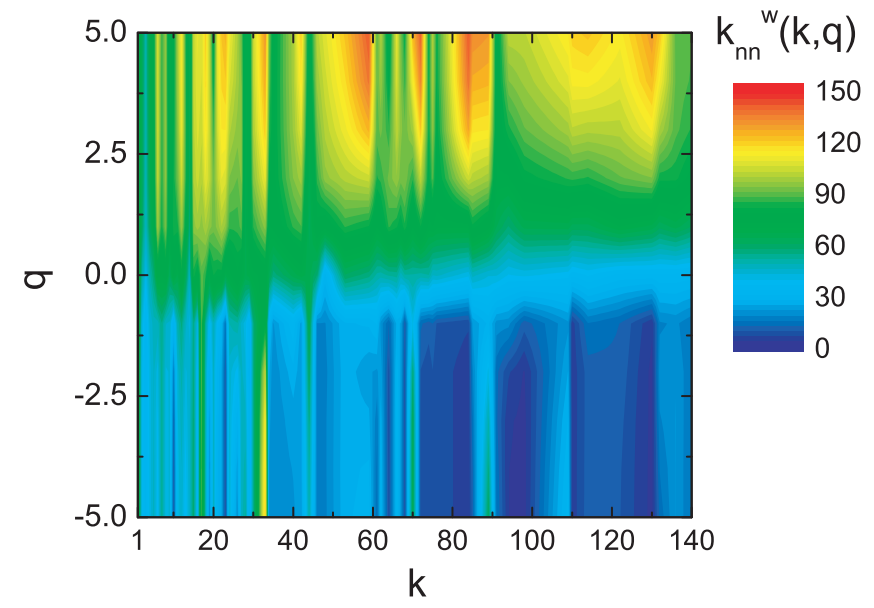

Figure 3: Metaweighted average nearest-neighbor degree of the USAN-I as a function of the degree $k$ and the parameter $q$.

is weakly disassortative in the region of $0<q \lesssim 3$, (4) $k_{n n}^{w}(k, q)$ does not depend on $k$ for $q \gtrsim 3$, and (5) $k_{n n}^{w}(k, q)$ is an increasing function of $q$ for any $k$. Considering the implications of these features, we can demonstrate how the degree correlation based on metaweights extracts meaningful information embedded in the USAN-I. Feature (1) implies the reasonable fact that airports weakly connected to hub airports (nodes with $k \gtrsim 100$ ) or middlescale airports (nodes with $50 \lesssim k \lesssim 100$ ) are very local airports (nodes with $k \lesssim 50)$. Feature $(2)$ is also explained by the same reason, namely, very local airports are connected to hub or middle-scale airports. Since all such connections are weak, the disassortative property is found only for $q<0$. Feature (3) is a consequence of the fact that airports connected to hub airports are hub or local airports but those connected to local airports are mainly hub airports. Feature (4) implies that airports very strongly connected to airport $i$ are hub airports independently of the scale of the airport $i$. This tendency is enhanced when increasing the strength of connections, which leads to feature (5). The metaweighted degree correlation analysis can extract all these reasonably understandable features of the USAN-I.

Figure 4 shows $k_{n n}^{w}(k, q)$ for the SCN. The profile of the MANND is totally different from that of the USAN-I. From the definition of $k_{n n}^{w}(k, q)$, it is obvious that $k_{n n}^{w}(k=1, q)$ does not depend on $q$, as seen in Fig. 4 (and in Fig. 3). In contrast to the case of the USAN-I, $k_{n n}^{w}(k, q)$ for the 


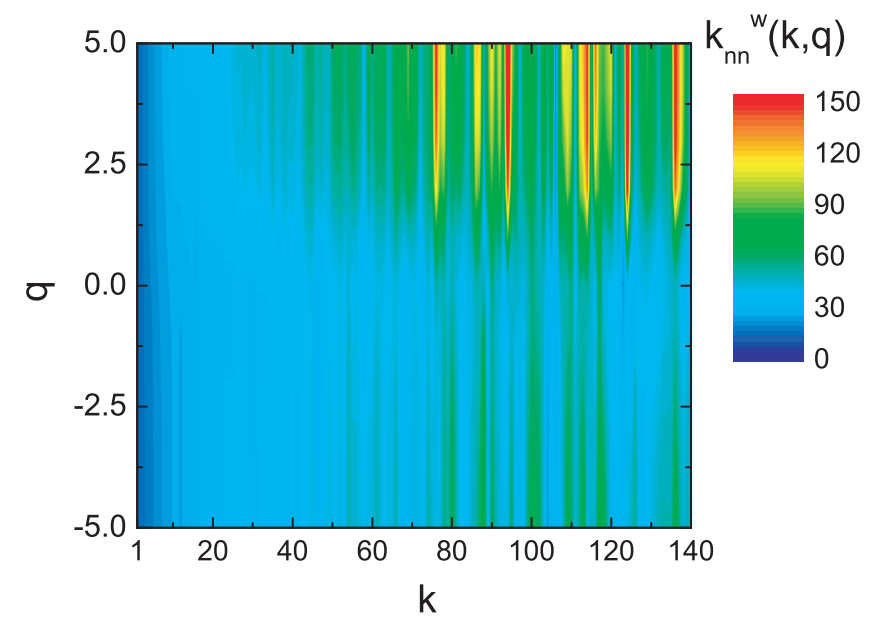

Figure 4: Metaweighted average nearest-neighbor degree of the SCN as a function of the degree $k$ and the parameter $q$.

SCN has a very weak $q$ dependence for $k \lesssim 50$. This is consistent with the previous result reported by Barrat et al. [8] in which the topological average nearest-neighbor degree [corresponding to $k_{n n}^{w}(k, q=0)$ ] is similar to the weighted average nearest-neighbor degree [corresponding to $k_{n n}^{w}(k, q=1)$ ] for an SCN different from the present one. Our result shows that the similarity between the topological and the weighted degree correlations holds even if very strong or very weak connections are enhanced. A possible interpretation of this property is that a young scientist with small $k$ collaborates either with researchers with large $k$ (his/her boss, for example) or small $k$ (his/her colleague in the same generation, for example). The number of papers coauthored by this scientist with each of the co-authoring partners is usually not related to the career of the partner (represented by degree $k_{n n}^{w}$ ). Thus, $k_{n n}^{w}(k, q)$ does not depend on $q$ for $k \lesssim 50$. After his/her growing up as a scientist $(k \gtrsim 50)$, some strategies of a professional researcher give a structure in $k_{n n}^{w}(k, q)$. The assortative property for $k \lesssim 50$ can also be related to the growth of a scientist. As the scientist's careers progress (increasing $k$ ), the co-authors also grow up (increasing $k_{n n}^{w}$ ). In the region of $k \gtrsim 50, k_{n n}^{w}(k, q)$ for $q>0$ exhibits the assortative property, whereas this quantity for $q<0$ is approximately 50 independently of $k$. The assortative property in the positive $q$ region implies that authors collaborating tightly with a big-name scientist are also big name. On the contrary, the $k$-independent profile of 
$k_{n n}^{w}(k \gtrsim 50, q<0)$ reflects the fact that an experienced scientist $(k \gtrsim 50)$ does not care about the careers or statuses of co-authors if the co-authoring relationship is only once or twice. We can observe relatively large fluctuations in the $k$ dependence of $k_{n n}^{w}(k, q)$ as in the case of $C^{w}(k, q)$. The origin of these fluctuations is the same as shown in Figs. 1(b) and 2(b) and it is not related to the intrinsic properties of networks. From these two examples, we conclude that the metaweighted degree correlation analysis extracts detailed information about weighted networks.

\section{Module analysis based on metaweights}

In this section, we show that the module analysis based on metaweights enables us to extract modules by systematically changing the importance of the topology and weights in the module decomposition by controlling the parameter $q$. Here, we extend the Girvan-Newman algorithm (GNA) [3, 7] for finding the module structures of weighted networks to a metaweighted version among many methods to decompose the network $[4,31,32,33,34$, $35,36,37,38,39,40,41]$. A weighted network that is singly connected and contains $N$ nodes is decomposed into small modules by the following scheme. First, we compute the edge betweenness centrality for the edge $i$ - $j$ defined as

$$
\sigma_{i j}=\sum_{i^{\prime}, j^{\prime}} \frac{b\left(i, j \mid i^{\prime}, j^{\prime}\right)}{b\left(i^{\prime}, j^{\prime}\right)}
$$

where $b(i, j)$ is the total number of shortest paths between nodes $i$ and $j$, and $b\left(i, j \mid i^{\prime}, j^{\prime}\right)$ is the number of them passing through the edge $i-j$. Then, we calculate the quantity $g_{i j}(q)=\sigma_{i j} / w_{i j}^{q}$, where the real continuous parameter $q$ is kept constant until the end of module decomposition. Next, we remove the edge with the largest $g_{i j}(q)$ from the network. The quantity $g_{i j}(q)$ is recalculated for the resultant network from which one edge is removed. This procedure is performed iteratively until the network is divided into two isolated subgraphs. During graph separation, we calculate the modularity defined by

$$
Q(q)=\frac{1}{2 W(q)} \sum_{i, j}\left\{w_{i j}^{q}-\frac{s_{i}(q) s_{j}(q)}{2 W(q)}\right\} a_{i j} \delta_{c_{i} c_{j}},
$$

where $W(q)$ is the total metaweight given by $W(q)=\sum_{i, j} w_{i j}^{q} / 2$, and $c_{i}$ is the index of the separated subgraphs including the node $i[6]$. This quantity 
increases if the separated subgraphs are well clustered. Then, we recalculate $g_{i j}(q)$ for the separated graphs and remove the edge with the largest $g_{i j}(q)$. This procedure is repeated until the network is completely decomposed into $N$ isolated nodes. The module structure of the initial network, based on a fixed value of $q$, is defined by the set of separated subgraphs giving the largest modularity $Q(q)$. The quantity $g_{i j}(q)$ is given by the ratio of the topological centrality $\left(\sigma_{i j}\right)$ to the weight-wise importance $\left(w_{i j}^{q}\right)$. By varying the parameter $q$, we can change this ratio systematically and obtain module structures under controlling the importance of the topology and weights. It should be emphasized that module structures obtained by the analyses with $q=0$ and $q=1$ are the same as those identified by the GNA for binary (topological) and weighted networks, respectively.

We apply the present module analysis based on metaweights to the US airport network (USAN-II) that is constructed from a data source that is different from that used for constructing the USAN-I [42]. In this network, 332 nodes correspond to airports in the United States, and weights are geographical distances between nodes connected by direct flights. Therefore, two geographically separated and topologically well-connected nodes in this network belong to the same module determined by the GNA. In fact, this tendency is observed in Fig. 5(a) which shows the result of the module analysis with $q=1$ for the USAN-II. In this figure, different colors represent different modules. We can find, however, that some of the nodes that are geographically close to each other belong to the same modules (same colors). This is because the strength of the topological connectivity between such nodes dominates the module-separation effect by small weights. In our analysis, one can enhance the role of weights in module decomposition by increasing the parameter $q$. Figure $5(\mathrm{~b})$ shows the result for $q=3$. In this result, nodes having the same color are located in geographically dispersed places. It is also possible to change the role of weights qualitatively by choosing a negative value of $q$. In the case of the USAN-II, edges connecting node pairs with short distances have large metaweights with a negative $q$. Thus, for $q<0$, an expected module structure of the USAN-II would be assembled by geographical clumps. Figure 6(a) indicates the module structure of this network obtained by setting $q=-1$. In contrast to the results shown in Fig. 5, modules in this result form regional blocks. The module structure identified by $q=-2$ is shown in Fig. 6(b). Since the role of weights in module decomposition becomes more important than that for $q=-1$, narrower regions are extracted as modules. In fact, single modules in Fig. 6(a) are 

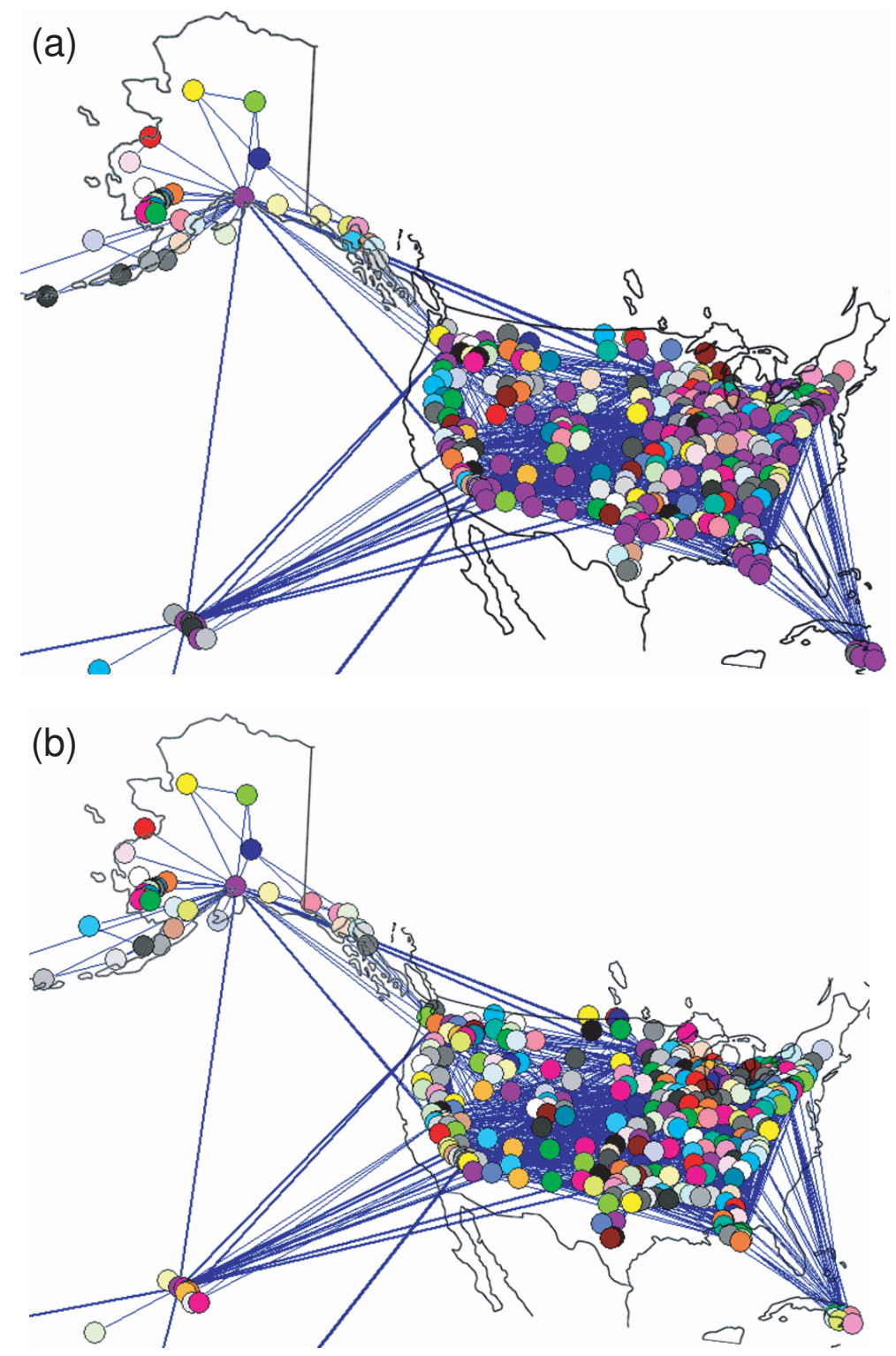

Figure 5: Module structures of the USAN-II determined by the present method with (a) $q=1$ and (b) $q=3$. Same colors indicate the same modules. The result (a) for $q=1$ is the same as that obtained by the GNA for weighted networks.

divided into several smaller modules in Fig. 6(b) [43]. These results indicate that (i) increasing $|q|$ Euclidean distances between airports are treated more 
importantly in the module decomposition of the USAN-II, (ii) by choosing negative $q$, we can extract modules in which airports are located close to each other in the Euclidean sense, and (iii) the module structures at $q=0$ and $q=1$ are the same as those identified by the GNA. In general, the module structure of a weighted complex network depends on how importantly we treat the weights as compared to the role of the topological connectivity. The present module analysis based on metaweights enables us to decompose a network by controlling the importance of the topology and weights in the decomposition procedure.

\section{Conclusion}

We have demonstrated that it is efficient to analyze statistical properties of weighted complex networks by introducing metaweights. In this paper, three examples of analyses based on metaweights have been argued, namely, the clustering coefficient, degree correlation, and module decomposition. The metaweighted clustering coefficient provides detailed information about the correlation between the strengths of edges and the tendency of triangle connections. An analysis of the degree correlation by using metaweighted nearest-neighbor degrees reveals that the attractive (or repulsive) tendency between hubs strongly depends on the strengths of edges being focused on. The module analysis based on metaweights makes it possible to decompose a weighted network by controlling the importance of roles of the topology and weight. In this paper, we analyzed rather trivial examples of real-world networks. It is interesting to apply module decomposition based on metaweights to some networks such as biological networks, in which the balance of the importance of weights and topology is not obvious. The metaweight analysis can also be extended to networks with weighted nodes (fitness). Considering the simplicity of the definition of metaweights and the efficiency of the metaweight analysis, further metaweighted statistical quantities other than the present three examples will provide considerable information about weighted complex networks.

\section{Acknowledgements}

Numerical calculations were performed on the HITACHI SR-11000 of Supercomputer Center, Institute for Solid State Physics, University of Tokyo. This work was supported in part by a Grant-in-Aid for Scientific Research by 

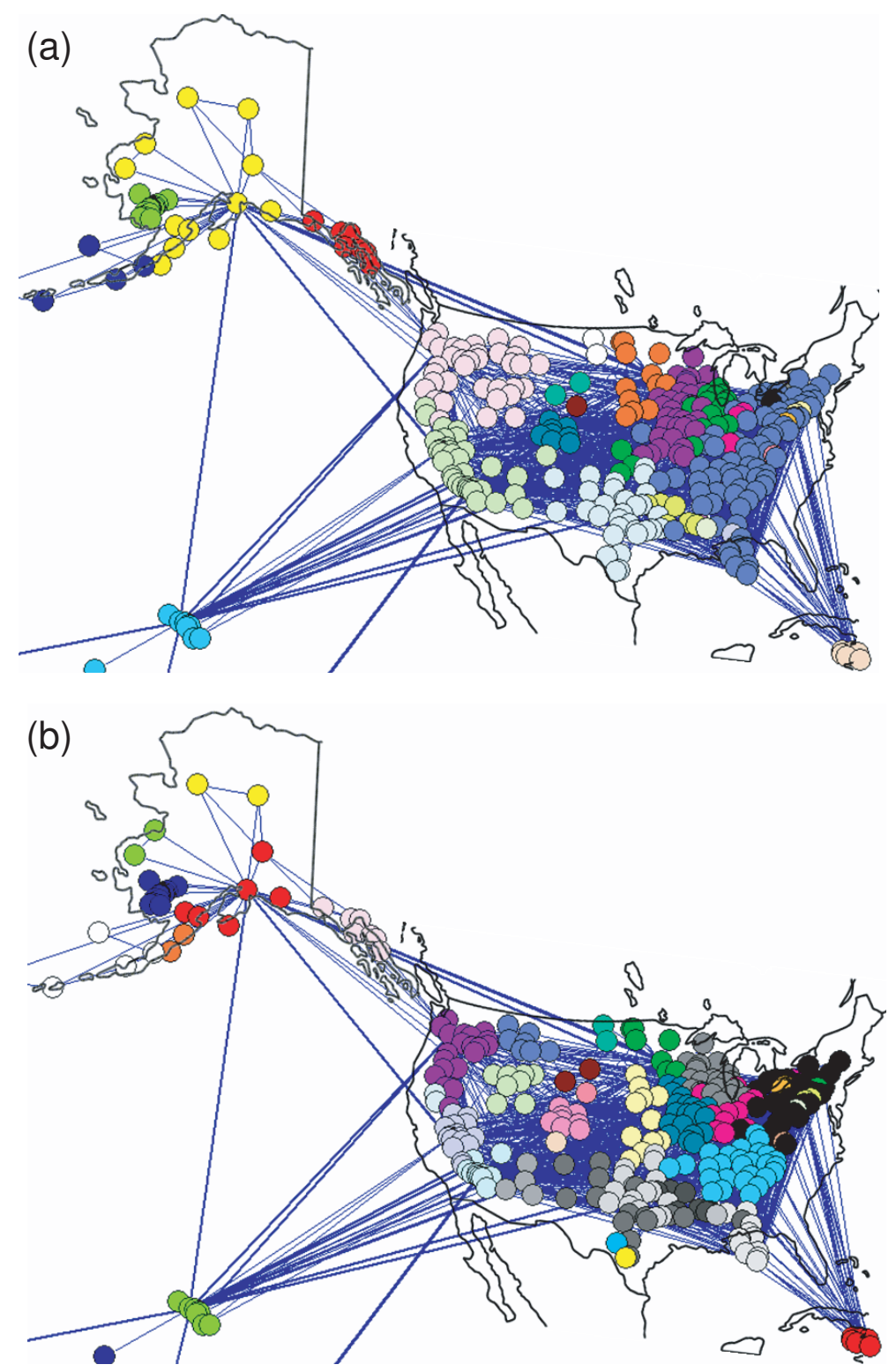

Figure 6: Module structures of the USAN-II determined by the present method with (a) $q=-1$ and (b) $q=-3$. Same colors indicate the same modules.

the Japan Society for the Promotion of Science (No. 19560001) and by the 21st Century COE Program entitled "Topological Science and Technology" 
from the Ministry of Education, Culture, Sport, Science and Technology of Japan (MECSST).

\section{References}

[1] D. J. Watts, S. H. Strogatz, Nature 393 (1998) 440.

[2] R. P.-Satorras, A. Vázquez, A. Vespignani, Phys. Rev. E 17 (2001) 258701.

[3] M. Girvan, M. E. J. Newman, Proc. Natl. Acad. Sci. USA 99 (2002) 7821.

[4] G. Palla, I. Derényi, I. Farkas, T. Vicsek, Nature 435 (2005) 814.

[5] I. J. Farkas, D. Ábel, G. Palla, T. Vicsek, New J. Phys. 9 (2007) 180.

[6] M. E. J. Newman, Phys. Rev. E 69 (2004) 066133.

[7] M. E. J. Newman, Phys. Rev. E 70 (2004) 056131.

[8] A. Barrat, M. Barthélemy, R. P.-Satorras, A. Vespignani, Proc. Natl. Acad. Sci. U.S.A. 101 (2004) 3747.

[9] S. H. Yook, H. Jeong, A.-L. Barabási, Y. Tu, Phys. Rev. Lett. 86 (2001) 5835 .

[10] S. Furuya, K. Yakubo, Phys. Rev. E 78 (2008) 066104.

[11] A.-L. Barabási, R. Albert, Science 286 (1999) 509.

[12] T. Zhou, G. Yan, B. H. Wang, Phys. Rev. E 71 (2005) 046141.

[13] S. N. Soffer, A. Vázquez, Phys. Rev. E 71 (2005) 057101.

[14] L. Tian, C.-P. Zhu, D.-N. Shi, Z.-M. Gu, T. Zhou, Phys. Rev. E 74 (2006) 046103.

[15] J. Saramäki, M. Kivelä, J.-P. Onnela, K. Kaski, J. Kertész, Phys. Rev. E 75 (2007) 027105.

[16] B. Zhang, S. Horvath, Stat. App. Genet. Mol. Biol. 4 (2005) 17. 
[17] P. Holme, S. M. Park, B. J. Kim, C. R. Edling, Physica A 373 (2007) 821.

[18] M. E. J. Newman, Phys. Rev. E 68 (2003) 026121.

[19] J.-P. Onnela, J. Saramäki, J. Kertész, K. Kaski, Phys. Rev. E 71 (2005) 065103(R).

[20] Network data Online at http://cxnets.googlepages.com/.

[21] V. Colizza, R. P.-Satorras, A. Vespignani, Nature Physics 3 (2007) 276.

[22] T. Opsahl, P. Panzarasa, Social Networks 31 (2009) 155.

[23] M. E. J. Newman, Proc. Natl. Acad. Sci. USA 98 (2001) 404.

[24] Network data Online at http://www-personal.umich.edu/ mejn/.

[25] M. E. J. Newman, Phys. Rev. E 64 (2001) 016132.

[26] M. E. J. Newman, Phys. Rev. Lett. 89 (2002) 208701.

[27] M. E. J. Newman, Phys. Rev. E. 67 (2003) 026126.

[28] C. C. Leung, H. F. Chau, Physica A 378 (2007) 591.

[29] H. Chang, B.-B. Su, Y.-P. Zhou, D.-R. He, Physica A 383 (2007) 687.

[30] M. Piraveenan, M. Prokopenko, A. Y. Zomaya, Eur. Phys. J. B 70 (2009) 275.

[31] M. E. J. Newman, Phys. Rev. E 74 (2006) 036104.

[32] J. Reichardt, S. Bornholdt, Phys. Rev. Lett. 93 (2004) 218701.

[33] J. Mei, S. He, G. Shi, Z. Wang, W. Li, New J. Phys. 11 (2009) 043025.

[34] W. Ren, G. Yan, X. Liao, L. Xiao, Phys. Rev. E 79 (2009) 036111.

[35] J. Duch, A. Arenas, Phys. Rev. E 72 (2005) 027104.

[36] M. Rosvall, C. T. Bergstrom, Proc. Natl. Acad. Sci. USA 104 (2007) 7327. 
[37] S. Zhang, R. S. Wang, X. S. Zhang, Physica A 374 (2007) 483.

[38] E. Estrada, N. Hatano, Appl. Math. Comp. 214 (2009) 500.

[39] Y. Hu, Y. Nie, H. Yang, J. Cheng, Y. Fan, Z. Di, arXiv:0902.3331.

[40] M. Tasgin, A. Herdagdelen, H. Bingol, arXiv:0711.0491.

[41] S. Fortunato, arXiv:0906.0612.

[42] V. Batagelj, A. Mrvar, (2006): Pajek datasets Online at http://vlado.fmf.uni-lj.si/pub/networks/data/.

[43] In this paper, we use the definition $\sigma_{i j}$ based on the topologically shortest paths, as shown in Eq. (5), instead of another possible definition based on the smallest metaweights. This is because the modularity of the decomposed USAN-II by the topological betweenness is larger than that by the metaweight-based betweenness. 